\title{
Object recognition using enhanced particle swarm optimization.
}

\author{
WILLIS, M., ZHANG, L., LIU, H., XIE, H. and MISTRY, L.
}

(C) 2020 IEEE. Personal use of this material is permitted. Permission from IEEE must be obtained for all other uses, in any current or future media, including reprinting/republishing this material for advertising or promotional purposes, creating new collective works, for resale or redistribution to servers or lists, or reuse of any copyrighted component of this work in other works. 


\title{
OBJECT RECOGNITION USING ENHANCED PARTICLE SWARM OPTIMIZATION
}

\author{
MICHAEL WILLIS ${ }^{1}$, LI ZHANG ${ }^{1}$, HAN LIU ${ }^{2}$, HAILUN XIE ${ }^{1}$, KAMLESH MISTRY ${ }^{1}$ \\ ${ }^{1}$ Department of Computer and Information Sciences, Faculty of Engineering and Environment, Northumbria University, \\ Newcastle, UK, NE1 8ST \\ ${ }^{2}$ College of Computer Science and Software Engineering, Shenzhen University, Shenzhen 518060, China \\ E-MAIL: michael.willis@northumbria.ac.uk, li.zhang@northumbria.ac.uk, han.liu@szu.edu.cn, hailun.xie@northumbria.ac.uk, \\ kamlesh.mistry@northumbria.ac.uk
}

\begin{abstract}
:
The identification of the most discriminative features in an explainable AI decision-making process is a challenging problem. This research tackles such challenges by proposing Particle Swarm Optimization (PSO) variants embedded with novel mutation and sampling iteration operations for feature selection in object recognition. Specifically, five PSO variants integrating different mutation and sampling strategies have been proposed to select the most discriminative feature subsets for the classification of different objects. A mutation strategy is firstly proposed by randomly flipping the particle positions in some dimensions to generate new feature interactions. Moreover, instead of embarking the position updating evolution in PSO, the proposed PSO variants generate offspring solutions through a sampling mechanism during the initial search process. Two offspring generation sampling schemes are investigated, i.e. the employment of the personal and global best solutions obtained using the mutation mechanism, respectively, as the starting positions for the subsequent search process. Subsequently, several machine learning algorithms are used in conjunction with the proposed PSO variants to perform object classification. As evidenced by the empirical results, the proposed PSO variants outperform the original PSO algorithm, significantly, for feature optimization.
\end{abstract}

Keywords:

Feature selection; Object recognition; Optimization

\section{Introduction}

Object recognition is an uprising research topic in computer vision. A great variety of techniques have been developed to undertake such recognition tasks, including machine learning and deep learning methods. Such classification tasks are challenging owing to the complexity of the real-world scenarios, which needs to be attended using specific techniques, e.g. image segmentation, background subtraction and feature selection. Machine learning methods have been widely used in human activity recognition, facial emotion recognition, autonomous driving. However, there are research gaps that can be identified in selecting the most effective feature subsets owing to complicated interactions between individual features as well as the premature convergence of the optimization methods when using machine learning algorithms to perform the recognition task. Therefore, in this research, we propose five Particle Swarm Optimization (PSO) variants with distinctive novel mutation and sampling iteration strategies to improve the performance of the original PSO model in feature selection. Besides that, several popular classification algorithms are employed to provide a comprehensive evaluation of the proposed feature optimization strategies.

\section{Literature review}

\subsection{Feature extraction}

Feature extraction is to employ feature descriptors to extract important features from the original data form which can be interpreted by subsequent algorithms. Owing to the extensive research focus in this specific area, many classical and effective feature extraction methods have been proposed [1]. As an example, Tian [2] extracted three main types of features for object recognition, including colour, texture and shape attributes, while Nixon and Aguado [3] extracted low-level features such as blobs, corners, edges and ridges for image classification. There are a number of popular existing feature extraction techniques, such as Local Binary Patterns (LBP), Histogram of Oriented Gradients (HOG), Haar-like features, and colour histograms. As a simple and computationally efficient feature descriptor, LBP takes a central pixel value and compares it with those of its neighbours. The LBP descriptor is able to capture edges, corners, lines, and spots, efficiently, in different application domains. 


\subsection{Feature selection}

Many evolutionary algorithms have been widely used in feature selection tasks owing to their outstanding search capability and simplicity to implement. Genetic Algorithm (GA) is one of the most popular evolutionary algorithms. In GA, a group of solutions/individuals is created which contains a collection of chromosomes. Each chromosome possesses a string of elements assigned between 0 and 1 , which in turn represent attributes in the problem domain. Crossover and mutation operations are employed to yield offspring solutions. Although GA has proven very successful in selecting appropriate features, it does have some limitations and drawbacks. The biggest issue, as indicated in Xue et al [4], is its high computational cost. The PSO model is another classical evolutionary algorithm. It is motivated by flocks of birds and schools of fish when they perform survival flocking behaviours. PSO employs a collection of swarm particles, which resembles the concept of the chromosomes in the GA. The particles move around the search space guided by the PSO search operation as they search for the most optimal solution. One of the major characteristics of PSO is that it follows the lead of the global and local best solutions to search for the global optimality [5-9].

\subsection{Classification}

Classification is the process of using machine learning algorithms to assign objects into categories based on their features and characteristics [10-11]. A classification algorithm learns what characteristics and features have in common for each class category.

Decision Tree (DT) [10] is a popular classification model which makes decisions by observing the threshold of attributes. In DT, a tree identifies the best split given the input data and adopts such an attribute as the top level of the tree. The similar process is repeated to identify lower level attributes and grow the tree. Naïve Bayes (NB) [10] classifier is a classifier that uses Bayes' theorem to perform the classification. NB obtains the probability of an object pertaining to a specific class by calculating the frequency of the attributes from the new object that have appearing within that specific class. K-Nearest Neighbours (KNN) [10] is one of the simplest classifiers and requires no additional input parameters other than the training data. In $\mathrm{KNN}$, an instance is assigned to the class most common among its neighbours. The $\mathrm{K}$ value simply represents the number of neighbours. Support Vector Machine (SVM) [10] is another popular method for classification by constructing a hyperplane that maximizes the margin between two classes. SVM maps the data into a different space using a kernel function to make data distribution more separable. Discriminant Analysis (DA) [10] is also widely used for data classification. Unlike linear classification algorithms, the mechanism of DA is to find the optimal solution that can best separate different classes. DA demonstrates many merits in handling classification tasks, e.g. low computational cost, efficient performance, and capability of solving multi-class classification. The aim of this study is to propose an effective PSO-based feature selection and benchmark the performance of five popular classifiers, i.e. DT, NB, KNN, SVM, and DA, for object recognition.

\section{The proposed PSO method for feature selection}

Despite effectiveness in tackling optimisation tasks and its simplicity [12-20], PSO suffers from premature convergence and local optima traps which severely affect its performance especially on real-life tasks with complex landscapes and multi-modal characteristics. In this study, five PSO variants are proposed to overcome the premature convergence and undertake the challenges of feature redundancy and interactions in the process of feature selection.

The first PSO variant, namely $\mathrm{IPSO}_{1}$, employs a mutation strategy for offspring generation to increase search efficiency and scope. Specifically, if a particle represents a selected feature subset, the mutation strategy is employed to generate a new solution by randomly flipping the scores of some elements between 0 and 1 . Therefore, there are features that are selected or un-selected by this mutation operation, in the newly generated offspring solution, in comparison with those of the original solution, to increase diversity. The mutation operation facilitates search scope and diversity owing to the fact that particles are able to include new features which can be possibly dismissed in previous iterations. As a result, the swarm has better chances of escaping from local optima traps and attaining the global optimality owing to the increased diversity and enhanced communication between particles.

Beyond the mutation strategy, a sampling iteration strategy is also proposed to further enhance search efficiency. Specifically, the learning mechanism of the PSO operation is not applied during the first several iterations. Instead, the particles are assigned various positions by using the mutation operation in randomly selected sub-dimensions. Their personal and global best experiences are subsequently recorded and updated accordingly. Once the search reaches a certain number of iterations, the particles are assigned with their corresponding personal best positions identified so far. Subsequently, the PSO search operation is applied for each particle for the remaining iterations for the search of the most optimal feature subsets. This sampling strategy enables the particles to explore the search space with strong starting 
points.

The number of sampling iterations can impose a great impact on the effectiveness of the proposed sampling strategy. Specifically, small sampling iteration settings are unlikely to make a difference while large settings constrain the power of evolution owing to the lack of evolving process. In this research, three novel PSO variants with different sampling iteration settings are employed, i.e. $\mathrm{IPSO}_{2}, \mathrm{IPSO}_{3}$, and $\mathrm{IPSO}_{4}$ $\mathrm{IPSO}_{2}$ and $\mathrm{IPSO}_{3}$ adopt five and ten iterations for sampling, respectively, while $\mathrm{IPSO}_{4}$ applies twenty iterations for sampling. With above settings, the impact of the sampling strategy can be thoroughly investigated.

The above idea can be further improved, as currently in the above search process, the particles are assigned with their corresponding personal best positions identified so far after sampling. However, the particles could also be assigned to positions that yielded the best overall fitness, in other words, assigning all the particles to the global best position or any other positions that led to the same fitness score. Therefore, we implement this strategy with ten sampling iterations in IPSO5. From such experimental settings, our experiments will be able to identify if it is better to use the personal or global best positions identified in the initial search as the starting points for subsequent search process. A summary of the proposed PSO variants is listed in Table 1.

TABLE 1. The summary of the proposed PSO variants

\begin{tabular}{|c|c|}
\hline $\mathrm{IPSO}_{1}$ & Mutation scheme $=$ yes \\
\hline $\mathrm{IPSO}_{2}$ & $\begin{array}{l}\text { Sampling iteration number }=5 \text {, } \\
\text { Each personal best solution is used after sampling, } \\
\text { Mutation scheme }=\text { yes. }\end{array}$ \\
\hline $\mathrm{IPSO}_{3}$ & $\begin{array}{l}\text { Sampling iteration number }=10, \\
\text { Each personal best solution is used after sampling, } \\
\text { Mutation scheme }=\text { yes. }\end{array}$ \\
\hline $\mathrm{IPSO}_{4}$ & $\begin{array}{l}\text { Sampling iteration number }=20 \text {, } \\
\text { Each personal best solution is used after sampling, } \\
\text { Mutation scheme }=\text { yes. }\end{array}$ \\
\hline $\mathrm{IPSO}_{5}$ & $\begin{array}{l}\text { Sampling iteration number }=10 \text {, } \\
\text { The global best solution is used after sampling, } \\
\text { Mutation scheme = yes. }\end{array}$ \\
\hline
\end{tabular}

\section{Experimental results}

In this study, a task of object recognition involving five most commonly seen items in home environments, i.e. chairs, tables, cupboards, lamps and clocks, is employed to evaluate the performance of the proposed PSO feature selection methods as well as the performance of five popular classifiers. All the images are extracted from an existing publicly available image database, where the images have clear backgrounds without cluttered scenes. However, the images have different sizes and scales. We employ 1250 and 500 images for training and test, respectively, in the subsequent experiments. Fig. 1 shows example database images.

Specifically, LBP is firstly used to undertake feature extraction. Then aforementioned five PSO variants are used for feature selection. Five classifiers are employed to perform classification, including NB, DA, KNN, SVM and DT. This study aims to gain insights into the performance of above five classifiers in undertaking object recognition and identify the most efficient one, as well as the efficacy of the proposed PSO methods. Each experiment is performed for multiple (i.e. 30) times and the mean results over a set of 30 runs are used for comparison to mitigate the effects of random factors. The experimental settings are as follows, i.e. population $=30$ and the maximum number of iterations $=500$.

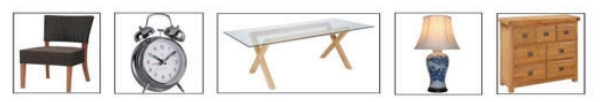

FIGURE 1. Example images of the employed data set

TABLE 2. The average results for DT

\begin{tabular}{ccccc}
\hline Algorithm & Fitness & $\begin{array}{c}\text { Overall Test } \\
\text { Accuracy }\end{array}$ & $\begin{array}{c}\text { Number of } \\
\text { Selected Features }\end{array}$ & $\begin{array}{c}\text { Run Time } \\
\text { (seconds) }\end{array}$ \\
\hline Non & 0.6808 & 0.7546 & 59 & - \\
\hline$P S O$ & 0.7740 & 0.8518 & 14.8 & 678.8564 \\
\hline $\mathrm{IPSO}_{1}$ & 0.7967 & 0.8771 & 14.4 & 731.5527 \\
\hline $\mathrm{IPSO}_{2}$ & 0.7978 & 0.8783 & 13.9 & 947.3814 \\
\hline $\mathrm{IPSO}_{3}$ & 0.7968 & 0.8769 & 14 & 669.4602 \\
\hline $\mathrm{IPSO}_{4}$ & 0.7939 & 0.8734 & 14.3 & 970.6640 \\
\hline $\mathrm{IPSO}_{5}$ & 0.7923 & 0.8703 & 11.6 & 676.3533 \\
\hline
\end{tabular}

\subsection{DT}

We first evaluate the proposed PSO models incorporated with the DT classifier using the test set. The DT model performed reasonably robust with the PSO-assisted feature selection. The average results over a set of 30 runs for each feature selection algorithm are shown in Table 2.

As shown in Table 2, all the proposed PSO variants achieve accuracy rates around $87 \%$. They demonstrate an overall advantage of 2\%-3\% in accuracy rates as compared with that of the original PSO algorithm. Moreover, most PSO variants select an average number of approximately 14 features, with $\mathrm{IPSO}_{5}$ identifying a slightly smaller feature size, i.e. 11.6. Also, all the algorithms vary greatly on the computational cost.

Moreover, the best performance is achieved by $\mathrm{IPSO}_{2}$, which uses five iterations for the initial search using the 
sampling strategy. The $\mathrm{IPSO}_{1}$ algorithm without the sampling mechanism achieves the best trade-off between performance and cost.

In terms of the PSO algorithms using the sampling strategy, the results indicate that the DT model benefits more from the models using the personal best positions as the strong starting points for the search process, than from the one (IPSO 5 ) using the global best position as the initial position. Besides that, $\mathrm{IPSO}_{3}$ uses the same number of sampling iterations as that of IPSO5. However, $\mathrm{IPSO}_{3}$ employs the personal best positions as the starting points for the search process and shows better capabilities in global exploration.

\subsection{DA}

We present the mean classification results over a set of 30 runs using the DA classifier in Table 3.

TABLE 3. The average results for DA

\begin{tabular}{ccccc}
\hline Algorithm & Fitness & $\begin{array}{c}\text { Overall Test } \\
\text { Accuracy }\end{array}$ & $\begin{array}{c}\text { Number of } \\
\text { Selected Features }\end{array}$ & $\begin{array}{c}\text { Run Time } \\
\text { (seconds) }\end{array}$ \\
\hline $\mathrm{Non}$ & 0.6461 & 0.7160 & 59 & - \\
\hline $\mathrm{PSO}$ & 0.7842 & 0.8686 & 41.7 & 1382.2649 \\
\hline $\mathrm{IPSO}_{1}$ & 0.8009 & 0.8873 & 42.3 & 1967.1143 \\
\hline $\mathrm{IPSO}_{2}$ & 0.8016 & 0.8878 & 39.9 & 1312.1273 \\
\hline $\mathrm{IPSO}_{3}$ & 0.8017 & 0.8881 & 41.9 & 1387.1074 \\
\hline $\mathrm{IPSO}_{4}$ & 0.7972 & 0.8832 & 43.5 & 1256.7030 \\
\hline $\mathrm{IPSO}_{5}$ & 0.7980 & 0.8839 & 41.5 & 1349.5117 \\
\hline
\end{tabular}

One distinctive observation with the results of DA is that the numbers of selected features are much higher than those using DT. Overall, the experiments from the DA model illustrate performance enhancement although the selected feature subsets are slightly larger. Besides that, all the PSO variants combined with the DA model obtain higher mean accuracy rates with an increase of around $2 \%$ as compared with that of the original PSO algorithm. Moreover, $\mathrm{IPSO}_{2}$, $\mathrm{IPSO}_{4}$, and $\mathrm{IPSO}_{5}$ demonstrate more superior performances, yet show less computational costs than those of the original PSO algorithm.

\section{3. $\mathrm{KNN}$}

We present the mean classification result over a set of 30 runs for each feature selection algorithm using the KNN classifier in Table 4. As shown in Table 4, both $\mathrm{IPSO}_{2}$ and $\mathrm{IPSO}_{3}$ achieve the (same) highest accuracy rate. In other words, the results indicate that there is no difference between using five and ten iterations for sampling when they are integrated with the $\mathrm{KNN}$ classifier. Also, all proposed algorithms outperform the original PSO model for feature optimization.

TABLE 4. The average results for $\mathrm{KNN}$

\begin{tabular}{ccccc}
\hline Algorithm & Fitness & $\begin{array}{c}\text { Overall Test } \\
\text { Accuracy }\end{array}$ & $\begin{array}{c}\text { Number of } \\
\text { Selected Features }\end{array}$ & $\begin{array}{c}\text { Run Time } \\
\text { (seconds) }\end{array}$ \\
\hline Non & 0.7001 & 0.7760 & 59 & - \\
\hline$P S O$ & 0.8556 & 0.9424 & 15.1 & 801.9560 \\
\hline$I P S O_{1}$ & 0.8752 & 0.9637 & 14.3 & 900.3082 \\
\hline $\mathrm{IPSO}_{2}$ & 0.8784 & 0.9691 & 17.1 & 885.0640 \\
\hline $\mathrm{IPSO}_{3}$ & 0.8784 & 0.9691 & 18.1 & 840.0837 \\
\hline $\mathrm{IPSO}_{4}$ & 0.8770 & 0.9669 & 16.3 & 765.3523 \\
\hline $\mathrm{IPSO}_{5}$ & 0.8743 & 0.9637 & 16.0 & 844.2961 \\
\hline
\end{tabular}

\subsection{SVM}

We illustrate the mean classification results over a set of 30 runs using the SVM classifier in Table 5.

TABLE 5. The average results for SVM

\begin{tabular}{ccccc}
\hline Algorithm & Fitness & $\begin{array}{c}\text { Overall Test } \\
\text { Accuracy }\end{array}$ & $\begin{array}{c}\text { Number of } \\
\text { Selected Features }\end{array}$ & $\begin{array}{c}\text { Run Time } \\
\text { (seconds) }\end{array}$ \\
\hline Non & 0.6425 & 0.7120 & 59 & - \\
\hline $\mathrm{PSO}$ & 0.7792 & 0.8544 & 10.4 & 7698.8672 \\
\hline $\mathrm{IPSO}_{1}$ & 0.8022 & 0.8787 & 9.3 & 8845.0516 \\
\hline $\mathrm{IPSO}_{2}$ & 0.7999 & 0.8765 & 9.3 & 7727.0202 \\
\hline $\mathrm{IPSO}_{3}$ & 0.7988 & 0.8760 & 9.9 & 7595.2729 \\
\hline $\mathrm{IPSO}_{4}$ & 0.7984 & 0.8748 & 9.5 & 6721.6580 \\
\hline $\mathrm{IPSO}_{5}$ & 0.7947 & 0.8711 & 9.7 & 7183.4308
\end{tabular}

In this experiment, the best performing algorithm is IPSO $_{1}$ with purely the mutation operation. Also both $\mathrm{IPSO}_{1}$ and $\mathrm{IPSO}_{2}$ select the smallest average number of features with around $2 \%$ performance improvement as compared with that of the original PSO model. However, IPSO ${ }_{1}$ does have a longer run time than those of the other methods, e.g. it is over 1118 seconds longer than the next largest run time of $\mathrm{IPSO}_{2}$. $\mathrm{IPSO}_{3}$ and $\mathrm{IPSO}_{4}$ have less run time than that of $\mathrm{IPSO}_{2}$. In other words, the sampling iterations have more impact on the computational cost than the performance. Also, all the proposed models have greater mean accuracy rates than that of the original PSO algorithm.

\subsection{NB}

We provide the mean classification results over a set of 30 
runs using the NB classifier in Table 6. Although the results using the NB classifier are less promising with low mean accuracy rates for all the search methods, all the proposed algorithms still outperform the original PSO model. The best result was achieved by $\mathrm{IPSO}_{2}$, which employs five iterations for sampling. However, this model has a far longer average processing time than those of other algorithms, i.e. it is over 405 seconds longer than the next highest average run time contributed by $\mathrm{IPSO}_{4}$. Interestingly the fastest algorithms are those with ten sampling iterations, i.e. $\mathrm{IPSO}_{3}$ and $\mathrm{IPSO}_{5}$. Also in this experiment, the IPSO5 algorithm that assigns the particles to a global best position as the starting points outperforms the $\mathrm{IPSO}_{3}$ model that uses the personal best solutions as the starting positions.

TABLE 6. The average results for NB

\begin{tabular}{ccccc}
\hline Algorithm & Fitness & $\begin{array}{c}\text { Overall Test } \\
\text { Accuracy }\end{array}$ & $\begin{array}{c}\text { Number of } \\
\text { Selected Features }\end{array}$ & $\begin{array}{c}\text { Run Time } \\
\text { (seconds) }\end{array}$ \\
\hline Non & 0.3658 & 0.4046 & 59 & - \\
\hline$P S O$ & 0.4808 & 0.5222 & 10.3 & 2469.2911 \\
\hline$I P S O_{1}$ & 0.4945 & 0.5351 & 8.3 & 2537.5662 \\
\hline$I P S O_{2}$ & 0.4949 & 0.5367 & 9.5 & 2965.0609 \\
\hline$I P S O_{3}$ & 0.4932 & 0.5343 & 8.8 & 2271.0346 \\
\hline$I P S O_{4}$ & 0.4942 & 0.5360 & 8.8 & 2559.7183 \\
\hline$I P S O_{5}$ & 0.4931 & 0.5347 & 9.3 & 2296.8273 \\
\hline
\end{tabular}

TABLE 7. Ranking of PSO variants on each classifier

\begin{tabular}{ccccccc}
\hline Classifier & $P S O$ & $\mathrm{IPSO}_{1}$ & $\mathrm{IPSO}_{2}$ & $\mathrm{IPSO}_{3}$ & $\mathrm{IPSO}_{4}$ & $\mathrm{IPSO}_{5}$ \\
\hline $\mathrm{DT}$ & $6^{\text {th }}$ & $2^{\text {nd }}$ & $1^{\text {st }}$ & $3^{\text {rd }}$ & $4^{\text {th }}$ & $5^{\text {th }}$ \\
\hline $\mathrm{DA}$ & $6^{\text {th }}$ & $3^{\text {rd }}$ & $2^{\text {nd }}$ & $1^{\text {st }}$ & $5^{\text {th }}$ & $4^{\text {th }}$ \\
\hline $\mathrm{KNN}$ & $6^{\text {th }}$ & $4^{\text {th }}$ & $1^{\text {st }}$ & $1^{\text {st }}$ & $3^{\text {rd }}$ & $4^{\text {th }}$ \\
\hline $\mathrm{NB}$ & $6^{\text {th }}$ & $1^{\text {st }}$ & $2^{\text {nd }}$ & $3^{\text {rd }}$ & $4^{\text {th }}$ & $5^{\text {th }}$ \\
\hline Average & $6^{\text {rd }}$ & $1^{\text {st }}$ & $5^{\text {th }}$ & $2^{\text {nd }}$ & $4^{\text {th }}$ \\
\hline
\end{tabular}

\subsection{Summary of results}

To obtain a better understanding of the comparison between the proposed different PSO models, their results are ranked by each classifier, as shown in Table 7. $\mathrm{IPSO}_{2}$ demonstrates the best feature selection performance across five different classifiers evidenced by the best ranking performance across different classification methods, followed by $\mathrm{IPSO}_{1}$ and $\mathrm{IPSO}_{3}$. Moreover, all five proposed PSO variants achieve more competitive feature selection performances as compared with those of the original PSO model for all the test cases.

\section{Conclusion}

In this research, we proposed five PSO variants which integrate mutation and sampling mechanisms to undertake the challenges of feature selection in object recognition. Five popular classifiers are investigated to evaluate their performance in object recognition tasks. The empirical results indicate that with the assistance of the proposed PSO-based feature selection, the classification performances are significantly enhanced with respect to all the classification models. Two of the proposed PSO methods demonstrate a significant superiority in feature selection among all investigated models, i.e. PSO variants employed five $\left(\mathrm{IPSO}_{2}\right)$ and ten $\left(\mathrm{IPSO}_{3}\right)$ sampling iterations, respectively, where the personal best solutions are used as the initial search positions after sampling. In future work, we will incorporate other repulsive search behaviours [17] and hybrid search strategies to further enhance the model efficiency. We will evaluate the proposed PSO models for other optimization tasks such as ensemble and deep learning model generation [20-28] to further enhance performance.

\section{References}

[1] G. Chandrashekar and F. Sahin, 2014. "A survey on feature selection methods," Computers \& Electrical Engineering, 40(1), pp.16-28.

[2] D. Tian, 2013. "A review on image feature extraction and representation techniques," International Journal of Multimedia and Ubiquitous Engineering, 8(4), pp.385-396.

[3] M. Nixon and A. Aguado, 2012. Feature extraction \& image processing for computer vision. Academic Press.

[4] B. Xue, M. Zhang, and W. Browne, 2014. "Particle swarm optimisation for feature selection in classification: Novel initialisation and updating mechanisms," Applied Soft Computing, 18, pp.261-276.

[5] K. Mistry, L. Zhang, S.C. Neoh, C.P. Lim, and B. Fielding. "A micro-GA Embedded PSO Feature Selection Approach to Intelligent Facial Emotion Recognition," IEEE Transactions on Cybernetics. 47 (6) 1496-1509. 2017.

[6] T. Tan, L. Zhang, S.C. Neoh, and C.P. Lim, C.P. "Intelligent Skin Cancer Detection Using Enhanced Particle Swarm Optimization," Knowledge-Based Systems. 2018.

[7] T. Tan, L. Zhang, C.P. Lim. B. Fielding, Y. Yu, and E. Anderson. "Evolving Ensemble Models for Image 
Segmentation Using Enhanced Particle Swarm Optimization," IEEE Access. 2019.

[8] T. Tan, L. Zhang and C.P. Lim. "Intelligent skin cancer diagnosis using improved particle swarm optimization and deep learning models," Applied Soft Computing, p.105725. 2019.

[9] W. Srisukkham, L. Zhang, S.C. Neoh, S. Todryk and C.P. Lim. "Intelligent Leukaemia Diagnosis with Bare-Bones PSO based Feature Optimization," Applied Soft Computing, 56. pp. 405-419. 2017.

[10] J. Han, M. Kamber, and J. Pei, 2011. "Data mining: concepts and techniques", $3^{\text {rd }}$ edn. Morgan Kaufmann.

[11] H. Liu and L. Zhang. "Advancing Ensemble Learning Performance through data transformation and classifiers fusion in granular computing context," Expert Systems with Applications. 2019.

[12] S.C. Neoh, L. Zhang, K. Mistry, M.A. Hossain, C.P. Lim, N. Aslam and P. Kinghorn. "Intelligent Facial Emotion Recognition Using a Layered Encoding Cascade Optimization Model," Applied Soft Computing. 2015.

[13] H. Xie, L. Zhang, C.P. Lim, Y. Yu, C. Liu, H. Liu and J. Walters. "Improving K-means clustering with enhanced Firefly Algorithms," Applied Soft Computing, 84, p.105763. 2019.

[14] L. Zhang, W. Srisukkham, S.C. Neoh, C.P. Lim and D. Pandit. "Classifier ensemble reduction using a modified firefly algorithm: An empirical evaluation," Expert Systems with Applications, pp.395-422. 2018.

[15] Y. Zhang, L. Zhang, S.C. Neoh, K. Mistry and A. Hossain. "Intelligent affect regression for bodily expressions using hybrid particle swarm optimization and adaptive ensembles," Expert Systems with Applications, 42 (22). pp. 8678-8697. 2015.

[16] S.C. Neoh, W. Srisukkham, L. Zhang, S. Todryk, B. Greystoke, C.P. Lim, A. Hossain and N. Aslam. "An Intelligent Decision Support System for Leukaemia Diagnosis using Microscopic Blood Images," Scientific Reports, 5 (14938). 2015.

[17] D. Pandit, L. Zhang, S. Chattopadhyay, C.P. Lim, and C. Liu. "A Scattering and Repulsive Swarm Intelligence Algorithm for Solving Global Optimization Problems," Knowledge-Based Systems. 2018.
[18] L. Zhang, K. Mistry, C.P. Lim and S.C. Neoh. "Feature selection using firefly optimization for classification and regression models," Decision Support Systems. 106 (2018) 64-85.

[19] L. Zhang, K. Mistry, S.C. Neoh. and C.P. Lim. "Intelligent facial emotion recognition using moth-firefly optimization," Knowledge-Based Systems. Volume 111, Nov. 2016, 248-267.

[20] Y. Zhang, L. Zhang and M.A. Hossain. "Adaptive 3D facial action intensity estimation and emotion recognition," Expert Systems with Applications, 42 (2015) 1446-1464.

[21] B. Fielding, T. Lawrence and L. Zhang. "Evolving and Ensembling Deep CNN Architectures for Image Classification," In IJCNN. 2019.

[22] L. Zhang and C.P. Lim. "Intelligent optic disc segmentation using improved particle swarm optimization and evolving ensemble models". Applied Soft Computing, 2020. p.106328.

[23] B. Fielding and L. Zhang. "Evolving Image Classification Architectures with Enhanced Particle Swarm Optimisation," IEEE Access, 6. pp. 68560-68575. 2018.

[24] T. Tan, L. Zhang and C.P. Lim. "Adaptive melanoma diagnosis using evolving clustering, ensemble and deep neural networks," Knowledge-Based Systems. 2019.

[25] P. Kinghorn, L. Zhang and L. Shao. "A Hierarchical and Regional Deep Learning Architecture for Image Description Generation," Pattern Recognition Letters. 2019.

[26] P. Kinghorn, L. Zhang and L. Shao. "A region-based image caption generator with refined descriptions," Neurocomputing. 272 (2018) 416-424.

[27] H. Xie, L. Zhang, and C. Lim. "Evolving CNN-LSTM Models for Time Series Prediction Using Enhanced Grey Wolf Optimizer", IEEE Access, 2020.

[28] B. Fielding and L. Zhang. "Evolving Deep DenseBlock Architecture Ensembles for Image Classification", Electronics, 2020. 